\title{
30 Years of Strong Interactions: a Three-Day Meeting in Honour of Joseph Cugnon and Hans-Jürgen Pirner
}

\author{
HLPW11: The Tenth Joint Meeting Heidelberg-Paris-Liège-Wroclaw
}

Published online: 13 June 2012

(C) Springer-Verlag 2012

The HLPW11 conference was held from the 6th to the 8th of April 2011 in Spa, a small Belgian town not far from Liège, renowned for its spring waters and its race track. It was organised by the Fundamental Interactions in Physics and Astrophysics Group of the University of Liège. This conference is the tenth of a series, which emanated from weekly seminars on the quark-gluon plasma, organised in Orsay by Dominique Vautherin in 1987-1988, and which was animated by a few physicists from Orsay and Saclay and by visiting scientists from the Universities of Heidelberg and Liège. At the end of the academic year, the participants came to the idea of setting regular meetings of physicists from the theory groups at the Universities of Orsay, Liège and Heidelberg and from the theory division at the Centre d'Etudes Nucléaires de Saclay. A few years ago, the organizers have decided to open progressively the participation to other institutions and recently the University of Wrocław in Poland joined the core of the organizing institutions, hence the current name and logo of the meetings.

The 2011 issue was organised to celebrate the recent retirement of two of the central figures of these conferences: Joseph Cugnon and Hans-Jürger Pirner. The topics discussed at the conference mainly concern areas in which they have been active and made important contributions: topics related to nuclear physics (spallation and the Liege intranuclear cascade, etc.), to hadronic physics (elastic scattering, nuclear matter effects), to QCD at zero temperature (exclusive reactions, deep inelastic scattering, transverse momentum distributions, Drell-Yan, etc.) and to the quark-gluon plasma and heavy-ion collisions (finite density, phase structure, etc.). Cross-links between these topics appeared in the discussions, witnessing the progress toward a increasingly coherent view of hadronic interactions. Other sessions have also reflected the evolution of the groups, and addressed astroparticle issues (cosmic rays and neutrinos). In view of the running of the LHC, part of the meeting was devoted to the related physics and its connection with cosmic rays. We hope that the reader will have the opportunity to appreciate this snapshot of many current aspects of hadronic physics, and that he or she will also appreciate the outlook on other issues provided by some of the contributors.

Acknowledgments On behalf of the Organizing Committee (J.R. Cudell, L. Favart, J.P. Lansberg, D. Mancusi, N. Matagne and S. Pedoux), we want to express our gratitude to the other members of the Scientific Advisory Committee (J. Cugnon, H.J. Pirner, H. Sazdjian, and L. Szymanowski) for their valuable suggestions in the preparation of the conference program. Our thanks also go to the speakers for the high quality of their presentations, to all the participants, who contributed to make this conference very lively, and to most of the contributors for their efforts to complete their manuscripts on time. We are especially grateful to

J. R. Cudell

IFPA, Université de Liège, Allée du 6 Aôut, 17, bât. B5, 4000 Liège, Belgium

J. P. Lansberg $(\varangle)$

IPNO, Université Paris-Sud, CNRS/IN2P3, 91406 Orsay, France

E-mail: lansberg@in2p3.fr

D. Mancusi

IRFU/SPhN, CEA Saclay, 91191 Gif-sur-Yvette Cedex, France 
the Fonds de la Recherche Scientifique-FNRS de la Communauté Française de Belgique for financial support. We also want to acknowledge the Interuniversity Attraction Pole "Fundamental interactions" from the Federal Science policy BELSPO, which allowed us to invite some of the speakers. We also want here to compliment the personnel of the Sol-Cress management for their help in the practical aspects of this event. 